\title{
La mediación docente para fomentar la educación emocional de los estudiantes de una Universidad Pública de Toluca
}

\section{Teacher mediation to promote emotional education for students of a Public University of Toluca}

\author{
LÓPEZ-BOTELLO, Felisa Yaerim †*, ROMERO-ROMERO, Araceli y GASCA-LEYVA, Michael E. \\ Universidad Autónoma del Estado de México, Facultad de Contaduría y Administración. Instituto Literario \# 100. C.P. 50000 \\ Col. Centro.
}

ID 1 ${ }^{\text {er }}$ Autor: Felisa Yaerim, López-Botello / ORC ID: 0000-0003-1732-497, CVU CONACYT ID: 596703

ID $1^{\mathrm{er}}$ Coautor: Araceli, Romero-Romero / ORC ID: 0000-0002-0328-0525, CVU CONACYT ID: 392910

ID $2^{\text {do }}$ Coautor: Michael E., Gasca-Leyva / ORC ID: 0000-0002-4906-5628

DOI: $10.35429 / J U P .2019 .7 .3 .27 .33$

Recibido: 15 de Enero, 2019; Aceptado 21 de Marzo, 2019

\section{Resumen}

La vida emocional es igual que las matemáticas y la lectura, puede manejarse con mayor o menor destreza y requiere un singular conjunto de habilidades (Goleman, 1995). El hecho de que los estudiantes sean capaces de controlar sus emociones reducirá los comportamientos problemáticos observados en los alumnos y a su vez mejorará sus relaciones sociales y familiares. La presente investigación servirá para comprender las causas de los malos comportamientos de los estudiantes, así mismo se propondrá a través de la mediación docente un taller "entre iguales" que permitirá mejorar el desempeño de los estudiantes, desarrollar emociones positivas, logrando un desarrollo integral. El diagnóstico fue dirigido a una muestra de 50 estudiantes de primer y segundo semestre de una Universidad Pública de Toluca, del turno matutino, elegidos por muestreo aleatorio simple. Así como a 20 habitantes de Toluca elegidos al azar; con la finalidad de saber cómo ellos perciben el comportamiento de los estudiantes de la misma Universidad. A través de 1 guía de observación y 2 cuestionarios estructurados, se identificó que debido a las situaciones que presencian y protagonizan los estudiantes manifiestan de manera inadecuada sus emociones, canalizándolas de manera negativa, lo que afecta gravemente su formación integral.

Mediación docente, inteligencia emocional, emociones positivas

\begin{abstract}
Emotional life is the same as mathematics and reading, it can be handled with greater or lesser skill and requires a unique set of skills (Goleman, 1995). The fact that students are able to control their emotions will reduce the problematic behaviors observed in students and in turn improve their social and family relationships. The present investigation will serve to understand the causes of the bad behaviors of the students, likewise it will be proposed through the educational mediation a workshop "between equals" that will allow to improve the performance of the students, develop positive emotions, achieving an integral development. The diagnosis was directed to a sample of 50 students of first and second semester of a Public University of Toluca, of the morning shift, chosen by simple random sampling. As well as 20 inhabitants of Toluca chosen at random; in order to know how they perceive the behavior of the students of the same University. Through an observation guide and 2 structured questionnaires, it was identified that due to the situations that students witness and protagonize, they manifest their emotions in an inadequate way, channeling them in a negative way, which seriously affects their integral formation.
\end{abstract}

Teaching mediation, emotional intelligence, positive emotions

Citación: LÓPEZ-BOTELLO, Felisa Yaerim, ROMERO-ROMERO, Araceli y GASCA-LEYVA, Michael E. La mediación docente para fomentar la educación emocional de los estudiantes de una Universidad Pública de Toluca. Revista de Políticas Universitarias. 2019. 3-7: 27-33.

\footnotetext{
* Correspondencia del Autor (Correo electrónico: fely_yaerim@ @otmail.com)

$\dagger$ Investigador contribuyendo como primer autor.
} 


\section{Introducción}

La inteligencia emocional es una facultad con la que todo ser humano debe de contar para lograr desempeñarse acertadamente dentro de la sociedad; desde tener una convivencia sana con los demás hasta conseguir un buen trabajo y lograr una estabilidad en todos los sentidos; así como el hogar; la escuela resulta ser un espacio predilecto para desarrollar este tipo de inteligencia y sin embargo las consecuencias de no hacerlo recaen en problemas como drogadicción, deserción escolar, violencia entre otros. La educación emocional es una innovación educativa que responde a necesidades sociales no atendidas en las materias académicas ordinarias. La fundamentación está en el concepto de emoción, teorías de la emoción, la neurociencia, la teoría de las inteligencias múltiples, la inteligencia emocional, los movimientos de renovación pedagógica, la educación para la salud, las habilidades sociales, las investigaciones sobre el bienestar subjetivo, etc.

\section{Justificación}

Según Bones, Perez, Rodriguez, Sanz, Borrell y Obiols, (2010), leídos en Ortuño, J, (2013) La encuesta Nacional de Salud en España reveló que entre los 10-20\% de los niños, adolescents y jovenes presentan algún tipo de trastorno mental o emocional.

De acuerdo a Blanchard, Gurka, y Blackman, 2006 leídos en Ortuño, J. (2013) un porcentaje elevado de niños, adolescents y jovenes puede presentar a lo largo de su vida dificultades en el ajuste psicológico, ocasionando un claro impacto, en las esferas personales, académicas, familiares y sociales, tales dificultades se inician en un $50 \%$ antes de los 15 años, por tal motivo las enfermedades físicas han disminuido notablemente pero por el contrario los problemas psicológicos han aumentado llegando a edades mayores.

Las emociones deben ser tomadas en cuenta dentro de la educación debido a que influyen en el razonamiento, en la toma de decisiones y en la conducta que presenta el individuo.
Según Cohen (2003) Aprender a leer en nosotros mismos y en las reacciones de los demás es tan importante como aprender palabras y cifras, es decir desarrollar la inteligencia emocional de los estidiantes de una Universidad Pública de Toluca para fortalecer sus emociones positivas y para canalizar las negativas resulta igual de importante que abarcar los contenidos temáticos que los programas de educación indican. La vida emocional es un ámbito que, al igual que las matemáticas y la lectura, puede manejarse con mayor o menor destreza y requiere un singular conjunto de habilidades (Goleman, 1995).

El hecho de que los estudiantes sean capaces de controlar sus emociones reducirá en gran escala los comportamientos problemáticos observados en los estudiantes y a su vez mejorará sus relaciones sociales y familiares. La presente investigación servirá a la comunidad educativa para comprender las causas de los malos comportamientos, así mismo permitirá mejorar el desempeño de los estudiantes de dicho nivel desarrollando la mayor cantidad de emociones positivas posibles logrando un desarrollo integral.

Se pretende a través de la mediación docente encontrar las técnicas y estrategias indicadas para la elaboración de un taller que permita fortalecer el desarrollo de las emociones positivas a través de la educación emocional.

Cabe plantear la siguiente pregunta de investigación:

¿De qué manera la mediación docente fomenta la educación emocional de los estudiantes, para lograr el desarrollo de sus emociones positivas en una Universidad Pública de Toluca?

Se parte del supuesto que, la educación emocional debe trabajarse desde el seno familiar y en todos los niveles educativos, los cuales son encargados de fortalecerla, sin embargo, en cada nivel se trabaja de manera distinta. 
La Educación emocional dentro de una Universidad Pública de Toluca puede trabajarse a través del taller sugerido en este trabajo el cual ofrece al docente las herramientas suficientes para fortalecer la inteligencia emocional en los estudiantes misma que influye de manera positiva en los estudiantes pues reduce los comportamientos violentos y enriquece sus capacidades adaptativas, además de que mejora el rendimiento escolar facilitando a los estudiantes la organización de tiempos y la estructuración de actividades.

El objetivo principal de la investigación es indagar acerca de cuál es la forma más adecuada para llevar a cabo la mediación docente y así fomentar la educación emocional con el fin de lograr el máximo desarrollo de las emociones positivas de los estudiantes de una Universidad Pública de Toluca.

Lo anterior, a partir de saber cuáles son los comportamientos negativos a los que incurren con mayor frecuencia los estudiantes de una Universidad Pública de Toluca. Reflexionar a los principales teóricos de mediación docente y los teóricos que consideran indispensable a la educación emocional en el desarrollo de los estudiantes; y analizar técnicas que permitan desarrollar las emociones positivas a través de la educación emocional, lo cual posibilitará mejoras en el desempeño de los estudiantes y permitirá su desarrollo integral.

\section{Partiendo de las emociones}

En Goleman (1995) La raíz de la palabra emoción es motere del latín "mover" y del prefijo "e" que implica alejarse. Lo que significa que en toda emoción hay una tendencia a actuar. De la misma manera Daniel y Michel Chabot (2009) señalan que la palabra emoción se deriva del verbo emocional, que significa poner en movimiento. A partir de lo anterior se puede mencionar que, las emociones son sensaciones producidas por una acción que conducen a reaccionar de manera positiva o negativa y que a su vez altera el estado de ánimo de los individuos.

Para lograr acrecentar el panorama a cerca de las emociones se retomó la postura del filósofo griego; Aristóteles. Para él las emociones son las afecciones del alma que se transmiten por el cuerpo, y como resultado de esto el cuerpo resulta afectado.
De acuerdo con Aristóteles leído en Goleman (1995); "la inteligencia emocional es la habilidad de ponerse furioso con la persona correcta, en la intensidad correcta, en el momento correcto, por el motivo correcto y de forma correcta". (1995, p.13)

El precursor del término educación emocional; Goleman (1995) enlista los beneficios a los que conduce la educación emocional, tanto para su comportamiento dentro y fuera de la escuela como su habilidad para aprender: Mayor tolerancia ante las frustraciones y el control de enojo, menor cantidad de bromas, peleas e interrupciones de la clase, mayor capacidad para expresar adecuadamente el enojo, sin pelear, menos suspensiones y expulsiones, menos comportamientos agresivos o autodestructivos, más sentimientos positivos sobre ellos mismos, la escuela, y la familia, mejor manejo del estrés.

Un punto importante de resaltar es que la mayoría de los aspectos retomados en la lista anterior interfieren con la atención prestada por el estudiante durante sus clases diarias, como mencionan en el libro Pedagogía Emocional, Daniel y Michel Chabot (2009) la atención es el elemento más sensible durante el proceso del aprendizaje, el cual se puede ver seriamente afectado por elementos cargados positiva o negativamente.

Muchas de las ventajas de la Educación Emocional recuperadas de la lista anterior se enfocan a lograr un mejor desempeño dentro del salón de clases y por ende un mayor aprendizaje.

Impulsar el desarrollo de las competencias emocionales es el fin de la educación emocional, Rafael Bisquerra (2009) las describe como "el conjunto de conocimientos, capacidades, habilidades y actitudes necesarios para tomar conciencia, comprender, expresar y regular de forma apropiada los fenómenos emocionales cuya finalidad es aportar un valor añadido a las funciones profesionales y promover el bienestar personal y social." (2009 p. 120). Fomentar las competencias emocionales en los estudiantes fortalecerá sus relaciones sociales en la escuela, contribuirá a lograr una mejor resolución de los conflictos lo que disminuirá notablemente la violencia y los problemas como adicciones, bajo rendimiento o deserción escolar. 
Las competencias emocionales se componen de cinco elementos ilustrados en la siguiente figura:

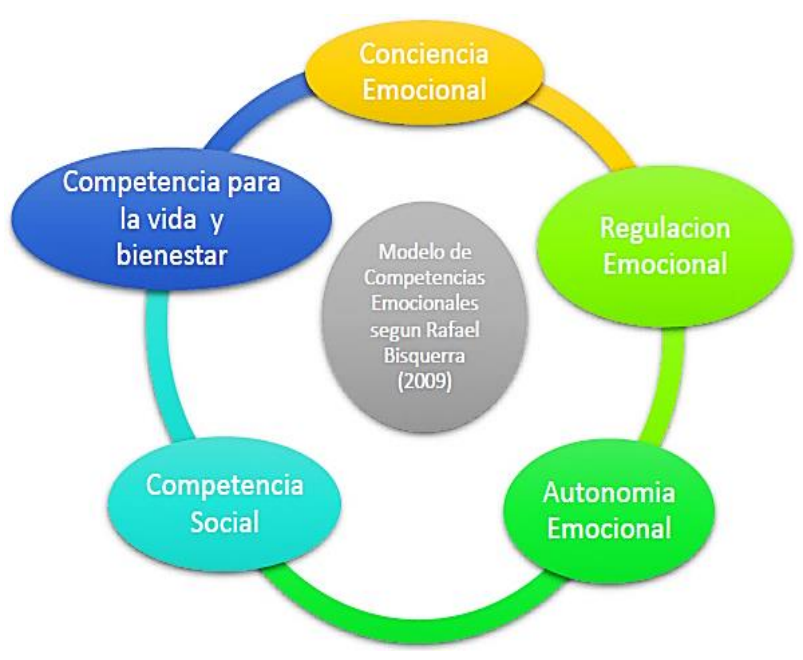

Figura 1 Competencias emocionales

Fuente: elaboración propia (2018), de acuerdo con Rafael Bisquerra (2009)

Johan Galtung, asigna al mediador como un trabajador del conflicto, también lo llama trabajador de la paz. El mediador menciona Galtung, busca generar un dialogo entre las partes que están en conflicto que esté basado en el criterio de legitimidad, es decir, en la idea de que somos parte del género humano, con el que nos hermanamos en sufrimiento. Desde ese lugar, agrega Galtung, existe responsabilidad en reducir la violencia y la destrucción. (Rozenblum, 2007).

De acuerdo con Galtung, la mediación aporta al conflicto conocimientos generales, habilidades, empatía, sin violencia, con creatividad, compasión y perseverancia, hasta encontrar la solución. Es imprescindible que el mediador, tenga un verdadero interés, deseos de aprender acerca de las partes al dialogar con ellas, intercambiando conocimientos generales y conocimientos locales. (Rozenblum, 2007).

\section{Metodología}

Partiendo de la investigación educativa, y con la firme intención de aportar más que explicaciones de carácter causal. La presente investigación logró una reflexión teórico- conceptual, se ubicó en el nivel descriptivo, debido a que se describió la conducta por parte de los estudiantes en el semestre agosto - diciembre 2018.
El diagnóstico fue dirigido a una muestra de 50 estudiantes de primer y tercer semestre de una Universidad Pública de Toluca, del turno matutino, elegidos por muestreo aleatorio simple. Así como a 20 habitantes cercanos a la Universidad de Toluca, objeto de estudio fueron elegidos al azar; con la finalidad de saber cómo ellos perciben el comportamiento de los estudiantes de la misma Universidad.

A través de 1 guía de observación y 2 cuestionarios estructurados, se identificó que debido a las situaciones que presencian y protagonizan los estudiantes manifiestan de manera inadecuada sus emociones, canalizándolas de manera negativa, lo que afecta gravemente su formación integral.

De acuerdo a lo expuesto anteriormente, el tipo de investigación que se realizó utilizó un enfoque cualitativo, que permitió describir e interpretar la información en su contexto real dentro de la universidad. El método elegido para resolver la problemática fue la InvestigaciónAcción.

Retomando a Latorre (2003), menciona que la Investigación-acción-educativa, es utilizada en la mayoría de los casos para describir una serie de actividades que realiza el profesorado en sus propias aulas con diferentes fines, tales como el desarrollo curricular, su autodesarrollo profesional, la mejora de los programas educativos, los sistemas de planificación, o la política de desarrollo.

El $55 \%$ de los estudiantes prefieren pensar antes de actuar; sin embargo, el 26\% prefiere golpear algo, por otra parte el $15 \%$ prefiere llorar y el $4 \%$ suele gritar a la personas; durante la observación realizada en el salón de clases, pasillos de la escuela, áreas verdes y cafetería se pudo apreciar el comportamiento de los estudiantes: se agreden físicamente, existe la burla, rechazo, gritos con palabras ofensivas a sus compañeros, abuso de poder.

Respecto a los maestros, se llegó a la conclusión que se mantienen las mismas características de una formación basada en el control, en la disciplina autoritaria, se deja entrever una relación de fuerza y dominación que los docentes ejercen en su labor educativa. 
El $59 \%$ de los estudiantes prefieren estar solos cuando están tristes, el $26 \%$ busca compañía, al $11 \%$ le dan ganas de llorar y únicamente el $4 \%$ se enoja con los demás.

El $37 \%$ de los alumnos piensa en sus cualidades cuando se siente vulnerable, el 33\% prefiere callar, el $19 \%$ se pone a la defensiva lo cual puede desencadenar en agresiones físicas o verbales y al $11 \%$ le dan ganas de gritar lo cual crea conflictos entre ellos.

De la misma manera el $90 \%$ de habitantes piensa que el lenguaje utilizado por los estudiantes de la universidad objeto de estudio no es el adecuado para su edad, su lenguaje incluye un gran número de groserías, palabras vulgares y ofensas, así mismo refieren que no les importa el lugar donde estén o la compañía que tengan. El $10 \%$ de habitantes menciona el lenguaje de los estudiantes es el adecuado, ya que son jóvenes y está acorde a su edad. Se tomó como base el método de investigación teórica histórico - lógico, se refirió a las tendencias y rasgos de las relaciones interpersonales entre estudiantes, a través de estrategias de mediación escolar. Al igual se aplicó el análisis y síntesis como método de investigación teórica, permitiendo puntualizar los objetivos de la investigación.

\section{Propuesta de Investigación}

De acuerdo a Melba Reyes, "el taller es una realidad integradora, compleja, reflexiva, en que se une la teoría y la práctica como fuerza motriz del proceso pedagógico"

Por medio de un taller dirigido a los estudiantes con 17 sesiones durante un semestre o intersemestral y con una duración cada sesión de 50 minutos.

El taller "entre iguales" se divide en las cinco esferas creadas por Peter Salovey: Conocer las propias emociones, manejo de emociones, propia motivación, reconocer emociones en los demás y manejar las relaciones; procurando fortalecer cada una de las etapas con las actividades sugeridas las cuales serán realizadas con ayuda de diversas técnicas como el juego, la lectura, el dibujo y las participaciones.
El taller se deberá llevar a cabo en diferentes espacios elegidos dependiendo de la actividad a desarrollar, por ejemplo, en las áreas verdes, en la biblioteca, en el salón asignado para taller o en el patio de la universidad. El objetivo del taller es fortalecer la Inteligencia Emocional de los estudiantes a través de la mediación docente, a través de la siguiente secuencia didáctica. Cada sesión tendrá su apertura, desarrollo y cierre.



LÓPEZ-BOTELLO, Felisa Yaerim, ROMERO-ROMERO, Araceli y GASCA-LEYVA, Michael E. La mediación docente para fomentar la educación emocional de los estudiantes de una Universidad Pública de Toluca. Revista de Políticas Universitarias. 2019 


\begin{tabular}{|c|c|c|}
\hline \multirow[t]{4}{*}{$\begin{array}{l}\text { Etapa 3. Propia } \\
\text { Motivación }\end{array}$} & $\begin{array}{c}\text { Sesión } \\
1 .\end{array}$ & $\begin{array}{l}\text { Objetivo de sesión 1: Invitar } \\
\text { al alumno a que reflexione } \\
\text { sobre las decisiones que ha } \\
\text { tomado a lo largo de su vida y } \\
\text { desarrollar su asertividad. }\end{array}$ \\
\hline & $\begin{array}{l}\text { Sesión } \\
2 .\end{array}$ & $\begin{array}{l}\text { Objetivo de sesión 2: Apoyar } \\
\text { al estudiante en la } \\
\text { organización de su tiempo y } \\
\text { administración de tareas para } \\
\text { reducir el estrés presentado } \\
\text { por los alumnos, resultado de } \\
\text { la presión escolar. }\end{array}$ \\
\hline & $\begin{array}{c}\text { Sesión } \\
3 .\end{array}$ & $\begin{array}{l}\text { Objetivo de sesión 3: } \\
\text { Favorecer la reflexión en los } \\
\text { estudiantes a cerca de lo que } \\
\text { han logrado y lo que quieren } \\
\text { lograr. }\end{array}$ \\
\hline & $\begin{array}{c}\text { Sesión } \\
4\end{array}$ & $\begin{array}{l}\text { Objetivo de sesión } 4: \\
\text { Impulsar al estudiante para } \\
\text { que recuerde el trayecto de } \\
\text { vida que ha tenido y sus } \\
\text { vivencias significativas. }\end{array}$ \\
\hline \multirow[t]{3}{*}{$\begin{array}{ll}\text { Etapa } & 4 . \\
\text { Reconocer } & \\
\text { Emociones } & \text { en } \\
\text { los demás } & \end{array}$} & $\begin{array}{c}\text { Sesión } \\
1 .\end{array}$ & $\begin{array}{l}\text { Objetivo de sesión 1: } \\
\text { Identificar el momento que } \\
\text { desencadena el sentir } \\
\text { determinada emoción. }\end{array}$ \\
\hline & $\begin{array}{l}\text { Sesión } \\
2 .\end{array}$ & $\begin{array}{l}\text { Objetivo de sesión 2: } \\
\text { Comprender que existen } \\
\text { diferentes maneras de saber } \\
\text { el estado emocional de una } \\
\text { persona, por su lenguaje } \\
\text { corporal, por sus palabras, o } \\
\text { por sus gestos. }\end{array}$ \\
\hline & $\begin{array}{c}\text { Sesión } \\
3 .\end{array}$ & $\begin{array}{l}\text { Objetivo de sesión } 3: \\
\text { Comprender la importancia } \\
\text { que tiene el lenguaje no } \\
\text { verbal para lograr el } \\
\text { reconocimiento de las } \\
\text { emociones de los otros. }\end{array}$ \\
\hline \multirow[t]{2}{*}{$\begin{array}{lr}\text { Etapa } & 5 . \\
\text { Manejar } & \text { las } \\
\text { relaciones } & \end{array}$} & $\begin{array}{l}\text { Sesión } \\
1 .\end{array}$ & $\begin{array}{l}\text { Objetivo de sesión 1: } \\
\text { Comprender los conflictos } \\
\text { que ha tenido el grupo y } \\
\text { destacar los buenos } \\
\text { momentos para fortalecer las } \\
\text { relaciones entre compañeros. }\end{array}$ \\
\hline & $\begin{array}{c}\text { Sesión } \\
2 .\end{array}$ & $\begin{array}{l}\text { Objetivo de sesión 2: } \\
\text { Conocer las relaciones } \\
\text { existentes en el salón de } \\
\text { clases para tomar decisiones } \\
\text { útiles para evitar conflictos. }\end{array}$ \\
\hline
\end{tabular}

Tabla 1 Etapas del Taller entre iguales Fuente: elaboración propia (2019)

\section{Resultados y Conclusiones}

Las emociones negativas predominan sobre las positivas en distintas circunstancias vividas por los estudiantes por ejemplo ante una mala calificación, ante un regaño o ante una pelea entre compañeros.
Así mismo se puede afirmar que el fortalecimiento de la Inteligencia Emocional es un trabajo en equipo entre padres, docentes y estudiantes, es necesario que entre estos tres agentes se forme un vínculo de comprensión y comunicación constante lo cual hará que los estudiantes se sientan comprendidos y muestren sus emociones abiertamente de la manera correcta.

Desarrollar una base emocional sólida en los estudiantes favorecerá al desarrollo de la asertividad, con ello serán capaces de tomar decisiones importantes como el rechazo de las drogas, seguir con sus estudios y resolver problemas. Los estudiantes requieren la aplicación de talleres que favorezcan su inteligencia emocional lo cual disminuirá el índice de peleas entre compañeros, violencia de género y agresiones.

Es importante prestar especial atención a los jóvenes para no atentar contra su personalidad, cuidar el lenguaje que utilizan los docentes con ellos y las estrategias utilizadas, procurando crear un ambiente de confianza, empatía y seguridad. La carencia de inteligencia emocional puede derivar en problemas conductuales que orillan al adolescente a tener un mal desempeño escolar.

La violencia escolar detectada en el diagnóstico es un problema latente que urge atender, y la educación emocional será un factor determinante para disminuirla.

La mediación implica la incorporación de habilidades de comunicación, de interrelación y de negociación. La escuela y las comunidades son los lugares más apropiados para incorporarla. La mediación promueve la asunción de responsabilidades, evitando hablar de culpas, ayuda a comprender que participar en un conflicto es significado de involucrarse en una mayor o menor proporción en la formación del mismo. La mediación, no está cumplimentada, hasta que los niños, jóvenes y adultos que participaron en el proceso hayan reflexionado o incorporado nuevas respuestas posibles ante una situación que genera un conflicto muy igual al que pueda producirse en un futuro. 


\section{Referencias}

Bisquerra, R. (2003). Educación emocional y competencias básicas para la vida: Revista de Investigación Educativa, Recuperado de: http://www.rafaelbisquerra.com/es/competencia s-emocionales/autonomia-emocional/81competencias-emocionales/100-conceptocompetencia-emocional.html el 21 de enero del 2019.

Cohen, J. (2003). La inteligencia emocional en el aula, Proyectos, estrategias e ideas. Argentina: Troquel.

Curiel, M. (2012) Modelo de atención a la violencia en el Estado de México.

Dávila, E. (2015). Orientación Educativa Para el Plan de Vida y Carrera en Estudiantes de Secundaria (Tesis de Licenciatura). México: Universidad Pedagógica Nacional 151.

Egg, A. (1999). Diagnostico Social, Conceptos y Metodología. Recuperado de http://datateca.unad.edu.co/contenidos/403021/ Bibliografia_/DIAGNOSTICO_SOCIAL_Maria_Jose_Aguilar_y_Ezequiel_Ander_Egg. pdf el 27 de febrero del 2019.

Estrada, P. (2009). Educación emocional: una necesidad de nuestro tiempo. Consideraciones sobre la importancia de la vida afectiva en la escuela hacia una formación integral del niño de primaria. (Tesis de Licenciatura). México: UNAM.

Goleman, D. (1995). La Inteligencia Emocional. México: Javier Vergara Editor.

Gómez, A. (1993). Ética Nicomaquea de Aristóteles. México: Porrúa.

Hernández, R. (2010). Metodología de la Investigación. México: Mc Graw Hill.

http://cedoc.inmujeres.gob.mx/ftpg/EdoMex/M EX_MA9_MODELO_VIOLENCIA_12.pdf el 20 de abril del 2018.

http://investigacionupn151.jimdo.com/tesis/ el 17 de marzo del 2019.
Latorre, A. (1996) "El Diario como Instrumento de Reflexión del Profesor Novel". Actas del III Congreso de E. F. de Facultades de Educación y XIV de Escuelas Universitarias de Magisterio. Guadalajara: Ferloprint.

López, L. (2014). Los ambientes afectivos: Una propuesta para disminuir la violencia simbólica en la escuela secundaria. (Tesis de Licenciatura). Universidad Pedagógica Nacional, Unidad 151. Toluca. Recuperada de Recuperado de:

Reyes, M.(2000). "El taller en el trabajo social. Taller de integración de teoría y práctica." Recuperado de http://acreditacion.unillanos.edu.co/CapDocente s/contenidos/NESTOR\%20BRAVO/Segunda\% 20Sesion/Concepto_taller.pdf el 04 de febrero del 2019

Rozenblum, S. (2007) Mediación. Convivencia y resolución de conflictos en la comunidad. Editorial GRAÓ, de IRIF, S.L. C/ Francesc Tárrega, 32-34. 08027 Barcelona. 